\title{
Neural Network Model for Performance Evaluation of Academic Staff of Tertiary Institutions
}

\author{
Okoye-Ubaka M.N. \\ Dept. of Computer Science \\ and Technology, \\ Bells University of \\ Technology, \\ Ota, Nigeria.
}

\author{
Adewole A.P. \\ Dept. of Computer \\ Science, \\ University of Lagos, \\ Akoka, \\ Nigeria.
}

\author{
Folorunso O. \\ Dept. of Computer \\ Science, \\ University of Agriculture, \\ Abeokuta, Nigeria.
}

\author{
Ezike, J.O.J. \\ Dept. of Information \\ Technology, \\ Bells University of \\ Technology, \\ Ota, Nigeria.
}

\begin{abstract}
This paper presents a neural network model for accomplishing the task of performance evaluation of academic staff of tertiary institutions. Data was collected from 10 randomly selected institutions using the completed Annual/Appraisal Performance and Evaluation Report (APER) form for academic staff. Fifteen Human Resource Metrics were considered which were classified under three main factors namely: Research, Teaching and Service. These are the major Human Resource foci of the Tertiary Institutions. The datasets were divided into three: train, validation and test data. The train data was presented to the Supervised Neural Network to approximate the fifteen Human Resource variables. The learning parameters for the training and testing of the survey data varied from 0.07 to 0.1 and the momentum parameter approached zero value (0.01 to 0.03$)$. Root Mean Square Error (RMSE) was computed for both the parametric models (Principal Component 0.80 and Factor Analysis 0.15). The result revealed that Multilayer Perceptron Neural Network (MPNN) with back propagation algorithm got better outcome when compared with those parametric models. Experimental results in this study demonstrated that MPNN based model can closely predict the Human Resource metrics, with minimum RSME at $90 \%$.
\end{abstract}

\section{General Terms}

Soft Computing, Artificial Intelligence, Human Resource Management.

\section{Keywords}

Neural Networks Model, Performance Evaluation, Tertiary Institutions, Factor Analysis, Principal Component Analysis.

\section{INTRODUCTION}

Whenever people gather to work, human resource issues become important. Human resource management is charged with integrating human resource planning with overall organizational planning, which includes career development, training and compensation. Others are performance evaluations, promotions, transfers and employee discipline. The performance profile of an employee is basically characterized by his biological data, academic and professional qualifications, area of specialization or generalization, history and work experience. The evaluation of employees of any organization is a subtask of human resource management. In other to accomplish the organization's goals and support its strategies, human resource objectives and strategies must be developed. The human resource objectives as identified in [1] are to: (a) Establish employee recruitment and selection systems for hiring the best possible employees consistent with the organization's needs.

(b) Maximize the potential of each employee to attain the organization's goal and ensure individual career growth and personal dignity.

(c) Retain employees whose performance helps the organization realize its goals and to relieve those whose performance is unsatisfactory.

(d) Ensure organizational compliance with state and federal laws that are applicable to the human resource management function.

Furthermore, HRM must be particularly sensitive to social and legislative forces as they impact on the organizations selection and development techniques. Certain factors such as nature of labour force, Federal and state legislation, labor unions and technological demands influence human resource management function. These factors also force organizations to re-examine their techniques for accepting or rejecting individuals for employment, transfer or promotion. Thus many organizations search and adopted suitable selection technique that can be demonstrated as valid, effective and practical for staffing decision. This is necessary because improper procurement and management of the human resources of an organization often leads to improper utilization of the other three assets (machine, money and time) and poor performance of organization in realizing her aim and objectives.

\subsection{Human Resource Assessment in Tertiary Institutions}

Tertiary institutions assets are man, money, machine and time. The man serving as the major driver of the other assets has three components, namely academic staff, administrative staff and technical staff. Appraisal of staff is done manually by the HRM in tertiary institutions. The appraisal process usually starts with assessment from each head of departments who also makes recommendations for the promotion of the staffs under his supervision. This recommendation is then passed to the Human Resource department, whose duty is to prepare minutes for each potential candidate. This will, in turn, be passed to the promotions committee. The committee finally implements the policies on selection and promotion matters. This process is depicted in Figure 1 below. However, in [2] it is believed that for HR to play its strategic role in any organization, Information Technology (IT) must be applied to strategic HR decisions like procurement, evaluation, compensation, and training and development. 


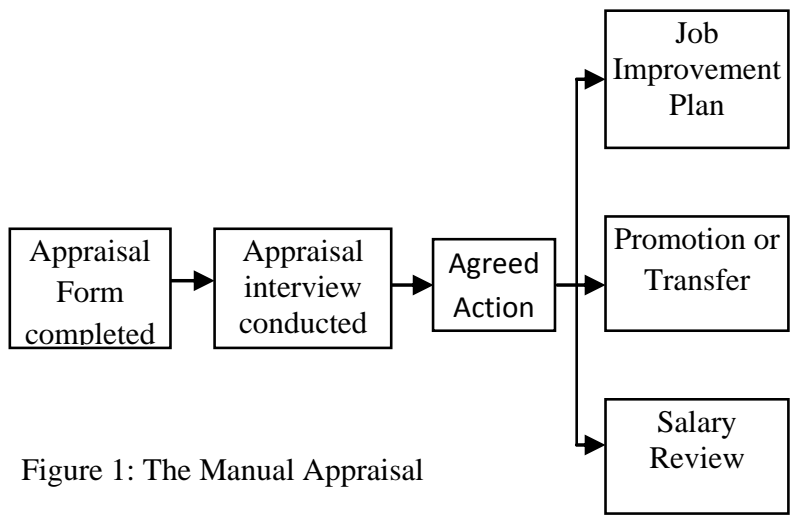

This research is motivated by the observed anomaly in the manual evaluation process which is bedeviled by the following shortcomings:

i. Inconsistent Reports: The manual procedure can produce inconsistent reports, in the sense that, an assessor who has the opportunity of evaluating a staff on two different occasions may not produce the same result.

ii. Lack of Standards: the manual procedure lacks standard, especially, when relating a staff of a division say ' $\mathrm{X}$ ' to a staff of a division say ' $\mathrm{Y}$ ' for a given position.

iii. Halo Effect

iv. Lack of good knowledge of the appraiser

v. Delay in decision making

vi. Partiality or Leniency

These shortcomings in the process result to decisions that are bias and full of sentiments.

Another reason that motives this research is the consideration of applying Neural Networks to resolve the observed manual shortcomings, particularly in view of Neural Networks ability to learn and adapt, which can be applied to resolve conflicts by collaboration, propagation and aggregation.

\section{LITERATURE REVIEW}

multiple criteria decision-making problems (MCDM) in different areas of life. It is therefore usually impossible to deal with a decision in terms of a single criterion. [3] Noted that not every evaluation technique is appropriate for a specific problem domain. Therefore, a suitable technique should be applied to particular situation to achieve our objectives.

The evaluation techniques are divided into two categories, the objective and the subjective methods. The objective technique evaluates decisions mostly based on numerical data, thus conclusion can be fairly supported without any ambiguity. It is, hence the subjective decisions that need to be objectified by means of multicriteria evaluating techniques. Weighing methods are commonly used in objectifying the subjective decision making problems in such a way that qualitative comparisons are quantified and ranked.

\subsection{Review of Conventional Evaluation Techniques}

Many evaluating techniques have been proposed to help decision makers to reach a sound conclusion. Some of the techniques described for the purpose of this research are:

\subsubsection{Analytic Hierarchy Process (AHP)}

This process is described in [4]. The primary concern of AHP is to simplify the complex evaluation problems into structural hierarchies. The major evaluating criteria are first identified, and consecutively subdivided into hierarchical levels. Items at the lowest level represent the alternatives that are to be compared and selected. Once the problem has been structured, the items or evaluating criteria at each level are pair wisely compared with quantitatively constructed positive reciprocal matrix. The corresponding eigenvector with respect to the principal eigenvalue of the matrix is determined and normalized. The normalized values imply the potency with which the various item in one level influence the items in the next higher level. Therefore, the relative impacts of the lowest level on the overall objectives can be computed.

\subsubsection{Delphi Hierarchy Process (DHP)}

The Delphi Hierarchy Process (DHP) is described in [4]. It is one of the systematic procedures used for extracting expert judgments. The Delphi approach typically involves three stages: (1) design of questionnaires, (2) the solicitation of group judgments and (3) the collation of responses. It is implemented by utilizing a panel with members in communication remotely through several rounds of questionnaires transmitted in writing.

\subsection{Review of Expert Systems for Evaluation of Performance}

The researchers in Artificial Intelligence (AI) for a long time have been looking for a way to develop and prototype intelligent computing systems that are capable of performing a variety of tasks that are difficult or impossible to do with conventional computing system.

Computer System that will operate intelligently like human brain requires the use of both quantitative and qualitative information as decision variables. The evaluation of human resource performance in cooperate organization is viewed in this research from two complementary angles. At one angle, the decision variables of human resource performance evaluation are considered to be related to one another in a manner similar to the structure and function of biological neural system. At the other angle, the value of some of the decision variables of human resource performance evaluation is considered to be fuzzy in nature. Fuzzy logic and neural network approaches to human resource performance evaluation is concerned with the evaluation of human performance and comparative analysis of the performance of peer personnel at various levels in corporate organization [5].

\subsubsection{Human Resource Evaluation System.}

As stated earlier, the conventional system of HR evaluation are mostly characterized by lack of standard, halo effect, lack of good knowledge of the appraiser, delay in decision making and leniency. This results to decisions that are bias and full of sentiments. In an attempt to overcome these problems, [2] proposed knowledge base system for the evaluation of the performance of human resource in the university environment. Their research attempted to carefully study the conventional method of human resource evaluation. Some existing works in the application of IT to human resource evaluation were studied with emphasis on the identification of methods of multicriteria decision analysis. A knowledge base system was developed for the evaluation of the performance of human resource in a University environment with emphases on academic staff component. The system was implemented in 
Visual basic 6.0 environment and integrates the filtering of both cognitive factors and emotional factors of human resource evaluation.

\section{METHODOLOGY}

Performance evaluation of staff of Tertiary institution requires numerical methods capable of analyzing dependence structure of a multivariate population. The performance evaluation variables are presented in Figure 2.

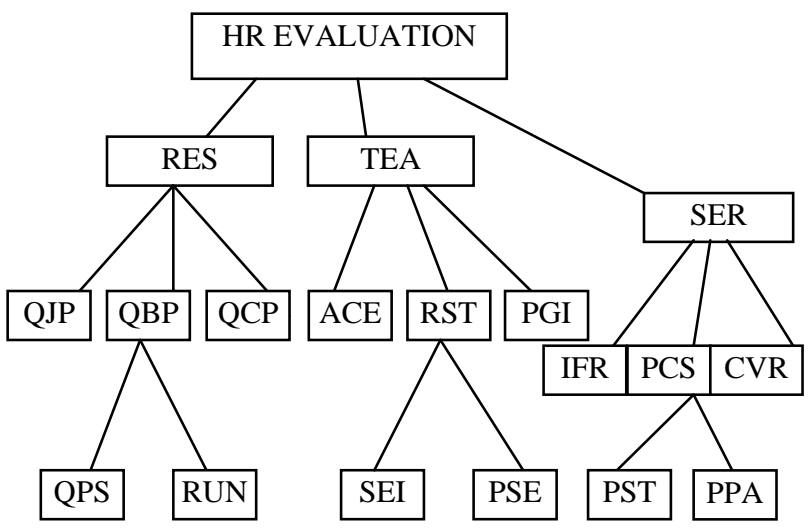

Figure 2: Human Resource Evaluation Model

RES - (Research), TEA - (Teaching), SER - (Service), QJP(Quality of Journal Publication), QBP - (Quality of book Publications), QCP - (Quality of conference Proceedings), QPS - (Quality of undergraduate Project Supervision), RUN(Relevance of Research to the needs of the University/ nation), ACE - (Academic experience), RST - (Relevance of Specialization to courses taught), SEI - (Students Evaluation of Instructor),

PSE - (Performance of Students in Examinations), PGI (Performance of graduates in the Industry), IFR - (Industrial Focus of Research), PST - (Performance of Students in Industrial Training), PCS - (Performance in Community Service), (Performance in Professional Associations), CVR (Commercial Viability of Research).

In this study, the above human resource management metrics were used as the data modeling tool for evaluating performance of academic staff based on three main factors, namely: research, teaching and service. These are the major investment foci of tertiary institutions.

Each are loaded on a number of the academic staff profile related decision variables and the loading on each factors are as follows:

\section{FACTOR 1- Research}

a. Quality of journal publication (QJP).

b. Quality of book publications (QBP).

c. Quality of conference proceedings (QCP).

d. Quality of undergraduate Project Supervision (QPS).

e. Relevance of research to the needs of the university /nation (RUN)

\section{FACTOR 2 - Teaching.}

a. Academic experience (ACE).

b. Relevance of specialization to courses taught (RST).

c. Students Evaluation of Instructor (SEI).

d. Performance of students in examinations (PSE).

e. Performance of graduates in the industry (PGI).

\section{FACTOR 3 - Service}

a. Industrial focus of research (IFR)

b. Performance of students in industrial training (PST).

c. Performance in community service $(P C S)$.

d. Performance in professional associations (PPA).

e. Commercial viability of research (CVR).

The three factors identified are interrelated and the performance of an academic staff in one factor could affect his performance in another factor. For instance, the performance in research and service could affect the teaching ability of the academic staff. This is because research exposes any academic staff to a lot of case materials, principles and practice of the subject matter. The effective participation in service exposes the teacher to the practical and contemporary realities of the field of study. Teaching could be regarded as the base factor because it is the primary assignment of any academic staff in the tertiary institution. However, a good teacher may not be a good researcher, and some research work may not have ready applications in the immediate community and industry.

The sources of the data for this study were selected randomly from ten (10) different institutions. What is important in the study is the proper evaluation of these performance variables and its statistical significance. Beyond the regression analysis within, that is classical ordinary least square (OLS) methods, a more contemporary methods multivariate and neural networks are employed.

\subsection{Multivariate Methods.}

Performance evaluation of staff of Tertiary institutions data set can be effectively analyzed through multivariate statistical tools due to their large dimensionality.

Multivariate methods are therefore effective tools due to their ability in clearly representing the multivariate structure of the data set achieving in the meantime the elimination of the contribution of the experimental error [6]. Among multivariate statistical analysis techniques, principal component analysis (PCA) and Factor analysis are adopted in the study.

\subsection{Principal Component Analysis (PCA)}

PCA is a multivariate pattern recognition method representing the objects, described by the original variables, into a new reference system given by new variables called principal components (PCs). [7], [8] and [6]. Each PC is calculated so that it explains in the maximum possible amount of residual variance contained in the original data set. The results of PCA provide two main tools for data analysis: The scores (that is the coordinates of the samples in the new reference system) and the loadings (that is the weights of the original variables on each PC). The analysis of score and loadings plots allows one to reach two main targets.

a. Identification of group samples showing a similar or opposite behavior and

b. Identification of the reasons for the similarities and diversities identified within the samples. 


\subsection{Factor Analysis}

The principal component analysis has been adopted as a technique for studying the dependence or correlational structure of the performance variables. To descry hidden factors which have generated the dependence or variation in the responses, factor analysis was adopted. Under the factor model each response variate are represented as a linear function of small number of unobserved able common-factor variates and a single latent specific variate. The common factors generate the covariances among the observable responses, while the specific terms contribute only to the variances of their particular responses. The mathematical form of the factor model must be one which generates the covariance or correlations among the responses. If that form is simple, and if the latent variates are few in number, a more parsimonious description of the dependence structure can be obtained

\subsection{Neural Networks Approach}

$\mathrm{NN}$ is used for processing in many real world problems, particularly where it is difficult to develop a programming algorithm. [9] The NN is trained by presenting examples to the network and adjusting it weight values accordingly by some learning rules until the $\mathrm{NN}$ output correspond acceptably to the desired output.

\subsubsection{Backpropagation Neural Network (BPNN).}

Backpropagation neural networks (BPNN) can approximate almost any function that has a finite number of discontinuities. Properly trained back propagation networks have proven to give responsible answers when presented within inputs that they have never seen [10]. Backpropagation adopted in this study guarantees that the order in which the patterns or set input/output are presented to the work does not influence the training.

A backpropagation network uses a supervised learning algorithm. An input pattern is presented to the network and then an output pattern is computed. This output pattern is compared to a target output pattern resulting in an error value. The error value is propagated backwards through the network and the values of the connection between the layers of units are adjusted in a way that the next time the output pattern is computed, it will be more similar to the target output pattern. This process is repeated until output pattern and target output pattern are almost equal.

\subsubsection{Fitting A Model To Data}

The rationale for the use of the neural network is forecasting or predicting a given target or output variable $Y$ from information on a set of observed input variables $X$. In order to make a good prediction in our model, we define a loss function $\mathrm{E}$ over the model parameters by using the dataset $D_{\mathrm{X}}$ $=\left(\mathrm{X}_{1}, \mathrm{X}_{2}, \ldots \mathrm{X}_{\mathrm{T}}\right)$ and targets $\mathrm{D}_{\mathrm{Y}}=\left(\mathrm{Y}_{1}, \mathrm{Y}_{2}, \ldots \ldots \mathrm{Y}_{\mathrm{T}}\right)$.

The study is interested in the distribution of a target $D_{y}$ given the values of the feature vector $D_{x}$ One suitable approach for modeling complex conditional densities $P\left(D_{y} \backslash D_{x}\right)$ is by tailoring the observed data as a sample from mixing density

$$
P\left(D_{y} \backslash D_{x}\right)=\sum_{j=1}^{n} g(\underset{j}{x}) P(y, X, j) \ldots \ldots \ldots \ldots(1)
$$

Where $\mathrm{g}_{\mathrm{j}}(\mathrm{x})$ is a conditional mixing proportion and can be regarded as a prior probability on the target $\mathrm{D}_{\mathrm{Y}}$ having been generated from the $\mathrm{j}^{\text {th }}$ component. The $\mathrm{P}(\mathrm{y} \backslash \mathrm{x}, \mathrm{j})$ are the component densities, generally taken from a simple parametric family. Here the data set is multinomial or Gaussian and the probability of generating a value y by component $\mathrm{j}$ is given as:

$P(Y, X, j)=\left(2 \pi r_{j}^{2}(x)\right)^{-1} \exp \left\{2 r_{j}^{2}(x)\right\}^{-1}\left(Y-\mu_{j}(x)\right) \ldots \ldots \ldots$

A frame work is proposed using factor analysis to take the various conditional parameters of the model, namely the means $\mu_{\mathrm{j}}(\mathrm{x})$ and the variances $\mathrm{r}_{\mathrm{j}}{ }_{\mathrm{j}}(\mathrm{x})$ and base each of them on general continuous and differentiable functions $f_{j}^{s}\left(X, \emptyset_{j}^{s}\right)$ in $X, S £\{\mu, r, g\}$ with parameter vector $\emptyset_{j}^{s} 2$. Here any function or approximator $\mathrm{f}_{\mathrm{j}}^{\mathrm{s}} \mathrm{(x)}$ that can be estimated using gradient information of the later derived cost function can be used, that is, non linear models or neural networks .

\subsubsection{Estimation}

Our goal of finding the best model is to find the values for the model parameters that minimize the error function $\mathrm{E}$, which provides us with an objective measure of predictions error for a specific choice of model parameters. By using backpropagation algorithm the error function $\mathrm{E}$ is given as

$$
\mathrm{E}_{\mathrm{f}}=(\mathrm{D}-\mathrm{X})(\mathrm{D}-\mathrm{X})^{\mathrm{T}} / 2 \ldots \ldots \ldots \ldots \ldots \ldots \ldots(3)
$$

Where $\mathrm{f}$ is forward, $\mathrm{T}$ indicates transpose; $\mathrm{D}$ is the desired output, $\mathrm{X}$ the actual output row matrices.

\subsubsection{Forecasting Models}

The model derived by the use of factor analysis was used as a guide in the training. The model selected was retained for output of sample estimation. The performance of the strategy is evaluated in term of the performance evaluation variables. Table 1 below is the correlation matrix of the sample data in a single file as generated using SPSS 16.0. The Correlation of the Performance Evaluation variables or inputs variables Dx can also be seen. The Correction analysis of the performance variables as in Table 1 revealed that there exists low correlation with each other.

Table 1: Correlation of the Performance variables

A principal component (PC) analysis is then used to maximize the sum of squared loadings of each factor extracted in turn. The $\mathrm{PC}$ analysis aims at constructing new variable $\left(\mathrm{P}_{\mathrm{i}}\right)$ called

\begin{tabular}{|l|l|l|l|l|l|l|l|l|l|l|l|l|l|l|l|}
\hline & QJP & QBP & QCP & QPS & RUN & IFR & PST & PCS & PPA & CVR & ACE & RST & SEI & PSE & PGI \\
\hline QJP & 1.000 & -.217 & -.301 & .011 & -.167 & .158 & -.108 & -.041 & .023 & .247 & .265 & .074 & .972 & .147 & -.138 \\
\hline QBP & -.217 & 1.000 & .255 & .057 & -.264 & .193 & .134 & -.143 & -.002 & .279 & .023 & -.123 & -.171 & .214 & -.025 \\
\hline QCP & -.301 & .255 & 1.000 & .011 & -.366 & .202 & .051 & .367 & -.002 & .417 & -.369 & .069 & .200 & -.028 & -065 \\
\hline QPS & .011 & .057 & .001 & 1.000 & .151 & -.014 & .123 & .043 & .148 & .200 & .100 & -.137 & .281 & .088 & -042 \\
\hline RUN & -.167 & -.264 & -366 & .151 & 1.000 & .051 & .064 & -.277 & -.129 & -.332 & .27 & .154 & .361 & -219 & -017 \\
\hline IFR & .158 & -.194 & -.202 & -.014 & .051 & 1.000 & .150 & -.193 & .199 & .045 & .181 & .125 & .207 & .178 & -031 \\
\hline PST & -.108 & .314 & .051 & .123 & .064 & .150 & 1.000 & -.077 & -.213 & .157 & .100 & .209 & .068 & -.127 & -.065 \\
\hline PCS & -.041 & -.143 & .367 & .043 & -.277 & .193 & -077 & 1.000 & -.013 & .313 & .018 & .033 & .165 & -.081 & -210 \\
\hline PPA & .023 & -.017 & -.002 & .148 & -.129 & .199 & -.213 & -.013 & 1.000 & -.093 & -.090 & -.250 & -.263 & .061 & .279 \\
\hline CVR & .247 & .279 & 417 & .200 & -.332 & .045 & .157 & .313 & -.093 & 1.000 & -.115 & .204 & .150 & .065 & -.099 \\
\hline ACE & .265 & .023 & -.369 & .100 & .027 & .181 & .100 & .018 & -.090 & -.115 & 1.000 & .215 & -.154 & .233 & -345 \\
\hline RSI & .074 & -.123 & .069 & -.137 & .154 & .125 & .209 & .033 & -.250 & .204 & .215 & 1.000 & .404 & .174 & -.164 \\
\hline SEI &.- .972 & -.171 & .200 & -.281 & .361 & .207 & .068 & .165 & -.263 & .150 & -.154 & .404 & 1.000 & .000 & -.125 \\
\hline PSE & .147 & .214 & -.028 & .088 & -.219 & .178 & -.127 & -.081 & .061 & .065 & .233 & .174 & .000 & 1.000 & -091 \\
\hline PGI & -.138 & -.025 & -.065 & -.042 & -.017 & -.031 & -065 & -.210 & .279 & -.099 & -.345 & -.164 & -.125 & -.091 & 1.000 \\
\hline
\end{tabular}


Principal Components out of a given set of variables $X_{j} s$ where $\mathrm{j}$ is $(\mathrm{j}=1,2,3 \ldots \ldots \mathrm{K})$. The variables with high loadings help in identifying the dimension of principal Component capturing. As the dimensions are independent, orthogonal rotation is used and when the sum of squared values of the loadings relating to the dimensions is taken, the sum is referred to as Eigenvalues or latent roots. Eigenvalue indicates the relative importance of each dimension for the particular set of variables being analyzed. The principal components with eigenvalue greater than 1 is taken for interpretation, given an $\mathrm{n} x \mathrm{p}$ matrix of performance evaluation variables, PC analysis reduced the number of columns. In this study $\mathrm{n}$ represents the number of objects for which performance evaluation metrics have been collected and $\mathrm{P}$ is the number of factors. The matrix is such that $\mathrm{n}=30$ and $\mathrm{P}=15$, thereby given an $30 \times 15$ matrix of multivariate data. Using PC analysis, the $\operatorname{nxp}(30 \times 15)$ matrix is reduced to $\mathrm{n} \mathrm{x} \mathrm{k}$ matrix (where $\mathrm{K}<\mathrm{P}$ ).

\subsubsection{Data Collection}

The datasets used for this research work were obtained randomly from ten (10) institutions. Each of the decision variables is given optional imprecise linguistic values, excellent, very good, good, average, or poor. The matrix of the weight attached to a linguistic value is shown in Table 2.

Table 2: The matrix of the weight attached to a linguistic value.

\begin{tabular}{|c|c|c|c|c|c|}
\hline $\begin{array}{c}\text { Linguistic } \\
\text { variable }\end{array}$ & Excellent & Very Good & Good & Average & Poor \\
\hline $\begin{array}{c}\text { Range of } \\
\text { values }\end{array}$ & $4.1-5.0$ & $3.1-4.0$ & $\begin{array}{c}2.1- \\
3.0\end{array}$ & $1.1-2.0$ & $0.0-1.0$ \\
\hline
\end{tabular}

SPSS generates the correlation matrix of the sample data in a single file as shown Table 1 above. The analysis of the correlation matrix shows that the highest correlation of 0.972 exists between the 'quality of undergraduate projects' (QUP) and 'performance of graduate in the industry' (PGI). The least correlation of 0.225 exists between 'quality of book publications' $(Q B P)$ and performance in professional associations' (PPA). The implication is that 'quality of undergraduate projects' is very likely to share the same factor with 'performance of graduates in the industry'. On the other hand, quality of book publications' is not likely to share the same factor with 'performance in professional associations'. The dataset comprised of three mainly factors, namely: teaching, research and service. The total data points collected were 330 in number. These data points were divided into 3 thus: train data (150), validation data (150), and test data (30). The information is presented in Table 3 below.

Table 3: Distribution of sample Data.

\begin{tabular}{|l|l|}
\hline TYPES Of DATASETS & SIZE Of DATASETS \\
\hline Train Data & 150 \\
\hline Validation Data & 150 \\
\hline Test Data & 30 \\
\hline Total & 330 \\
\hline
\end{tabular}

Table 4: Weight of Decision Variables

\begin{tabular}{|c|c|c|c|}
\hline $\begin{array}{c}\text { MATCH } \\
\text { PARAMETER }\end{array}$ & POINT & $\begin{array}{l}\text { TOTAL } \\
\text { POINT }\end{array}$ & WEIGHT \\
\hline Age & 01 & \multirow{4}{*}{04} & \multirow{4}{*}{0.0482} \\
\hline Sex & 01 & & \\
\hline State of Origin & 01 & & \\
\hline Marital Status & 01 & & \\
\hline $\begin{array}{l}\text { Academic } \\
\text { Qualification }\end{array}$ & 10 & \multirow{3}{*}{26} & \multirow{3}{*}{0.3133} \\
\hline Class & 10 & & \\
\hline Subject & 06 & & \\
\hline $\begin{array}{l}\text { Professional } \\
\text { Qualification }\end{array}$ & 02 & \multirow[t]{2}{*}{03} & \multirow[t]{2}{*}{0.0361} \\
\hline Status & 01 & & \\
\hline Relevant Experience & 05 & \multirow{4}{*}{20} & \multirow{4}{*}{0.2410} \\
\hline $\begin{array}{l}\text { Current } \\
\text { Responsibility }\end{array}$ & 05 & & \\
\hline Relevant projects & 05 & & \\
\hline Tools Used & 05 & & \\
\hline Psycho-metric Test & 30 & 30 & 0.3614 \\
\hline Total & 83 & 83 & 1.0000 \\
\hline
\end{tabular}

\section{[11]}

The train data that serves as input data, 150 in size, was presented to Neural Network for evaluation performance of the academic staff in batch mode with the feedforward architecture and backprogation algorithm. The training was done on a supervised manner. The supervised training is a form of training method whereby there is one or more target values which the network is meant to approximate. Each of the 150 data points were compressed into 15 data points by adding the academic staff profile related decision variables, the loading on each factors and weight of decision variables (Table 4). This preprocessing was done using Microsoft Excel.

The first sets of data (train data) were presented along with other two datasets (validation and test data). These last two datasets were used to simulate the network so as to see the generalization power of the network when introduced to the data it has not been previously exposed to. In order to discover the most appropriate topology for the network, 15 sets of topologies for each of the 3 training functions (45 topologies altogether) were used in the network one after the other.

The network used in this research work belongs to the Multilayer Feed Forward networks. Feed- Forward networks are used in situations where all information is taken at once. In this type of neural network, the data flows through the network in one direction, and the answer is based solely on the current set of inputs. The input nodes are connected to every node of the hidden layer but are not directly connected to output node. Thus the network does not have any lateral or shortcut connections [12]. Neural network repetitively adjusts different weights so that the difference between desired output from the network and actual output from neural network is minimized. The network learns by finding a vector of connection weights that minimizes the sum of squared errors on the training data set. The training set contains two-thirds of the collected data.

The network was trained using the backpropagation algorithm. The total data points collected were 330 
observations, 150 are used for the training set, 30 for the test data and 150 for the validation set. Neural networks software supplies corresponding predicted values for the observations of the validation set and the coefficient of determination.

Table 5: The values of the Performance Coefficient and their test Model

\begin{tabular}{|l|l|l|l|l|}
\hline Coefficient & VALUE & $\begin{array}{l}\text { SE(standard } \\
\text { error) }\end{array}$ & T VALUE & $\operatorname{Pr}(>\mid \mathbf{t} \mathbf{|})$ \\
\hline Intercept & 4.7695 & 1.879 & 2.5378 & 0.0227 \\
\hline QJP & -0.0687 & 0.0802 & -0.8562 & 0.4054 \\
\hline QBP & -0.0580 & 0.1534 & -0.3780 & 0.7107 \\
\hline QCP & -0.1423 & 0.0787 & -1.8079 & 0.0907 \\
\hline QPS & 0.2514 & 0.1028 & 2.4457 & 0.0273 \\
\hline IFR & -0.1024 & 0.1069 & -0.9586 & 0.3530 \\
\hline PST & -0.0223 & 0.0978 & -0.2279 & 0.8228 \\
\hline PCS & -0.2979 & 0.2195 & -1.3574 & 0.1947 \\
\hline PPA & -0.0199 & 0.0715 & -0.2777 & 0.7850 \\
\hline CVR & -0.0929 & 0.1015 & -0.9152 & 0.3746 \\
\hline ACE & 0.0025 & 0.0515 & 0.0483 & 0.9621 \\
\hline RST & 0.0648 & 0.1587 & 0.4084 & 0.6887 \\
\hline SEI & 0.4508 & 0.1467 & 3.0730 & 0.0077 \\
\hline PSE & -0.1189 & 0.0910 & -1.3064 & 0.2111 \\
\hline PGI & -0.0321 & 0.1081 & -0.2974 & 0.7703 \\
\hline
\end{tabular}

validation data.

Table 6: Shows the MSE, $\mathrm{R}^{2}$ and R.

\begin{tabular}{|l|l|}
\hline MSE & 0.4003 \\
\hline$R^{2}$ & 0.661 \\
\hline$R$ & 0.813 \\
\hline
\end{tabular}

The correlation of the predicted change and observed change is represented by the coefficient of correlation $R$. The significant level of a validation is indicated by a P-value. A commonly accepted $\mathbf{P}$-value is $\mathbf{0 . 0 5}$.

\section{SYSTEM IMPLEMENTATION AND PERFORMANCE EVALUATION}

In this research work neural network (NN) is implemented using this mathematical model:

$n e t_{k}=X_{1} W_{k 1}+X_{2} W_{k 2}+\ldots+X m_{m} W_{k m}=\sum \mathbf{x}_{\mathbf{i}} W_{k i} \quad$ Adapted from [13].

First, there are several inputs $\mathbf{X i}$, where $\mathbf{i}=\mathbf{1}, \mathbf{2} \ldots \mathbf{m}$. Each input $\mathbf{X i}$ is multiplied by the corresponding Weights wki where $\mathbf{k}$ is the index of a given neuron in an NN. A simple two hidden layers BP-NNs structure is show in Figure 3. Typical two hidden layers BP-NNs have one input layer, two hidden layers and one output layer. And it has five steps of execution: Initialization, Forward Computation, Backward Computation, Weight value update and Iteration. In the step of initialization, we need initialize the parameters of $\mathbf{w}_{\mathrm{ij}}$ and $\boldsymbol{\theta}$, where wij is the synaptic weight that corresponds to the connection from neuron unit $\mathbf{i}$ to $\mathbf{j}$ and $\boldsymbol{\theta}$ is bias of a neuron.

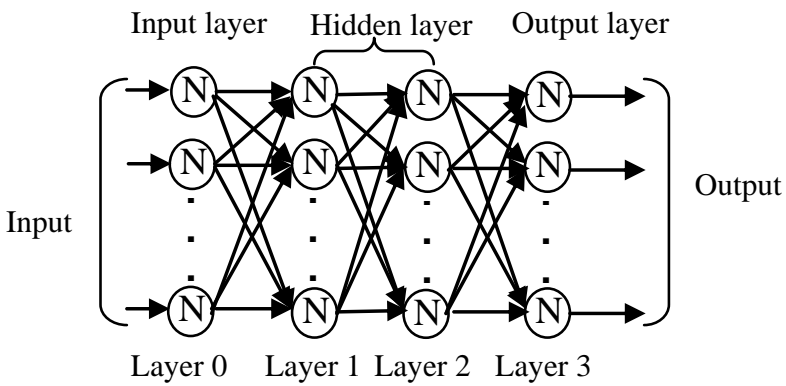

Figure 3 Structure of Two hidden layers BP-NNs

In Forward Computation step, the function is:

$$
\begin{aligned}
& \text { net }_{i}=\sum w_{i j} o_{j}+\theta \\
& o_{i}=f\left(\text { net }_{i}\right) \\
& f(x)=1 /\left(1+e^{-1}\right)
\end{aligned}
$$

$n e t_{i}$ is a weighted sum of this layer; $\mathrm{o}_{j}$ is an output of the preceding layer; $f(x)$ is an unipolar sigmoid function for the activation function.

In Backward Computation step, function for output layer is:

$$
\delta=f^{\prime}\left(\text { net }_{i}\right)\left(y_{i}-o_{i}\right)
$$

For hidden layers, function is:

$$
\delta=f^{\prime}\left(\text { net }_{i}\right) \sum_{k} \delta_{k} w_{k j} \ldots
$$

$\delta$ is a local gradient for the neuron and $y_{i}$ is a teaching single.

In Weight value update step, function is as follows:

$$
w_{i j}(t+1)=w_{i j}(t)+\eta \delta_{i} o_{j}
$$

Where $w_{i j}(t+1)$ is the update weight value and $\eta$ is learning rate.

In Iteration step, the Forward and Backward Computations for each training example are repeated. When training is completed, the Forward Computation is carried out. The network with specified weights can be used for testing a set of data different than those used for training. The result achieved is used for generalization of the approximation of the network.

\subsection{Implementation of Gradient Descent Algorithm}

There are two ways of implementing the above visa: incremental and batch modes. The incremental mode is the method whereby the gradient is computed and the weights updated after each input in the input vectors is applied to the network. On the other hand, the batch mode (which is used in this research work)updates weights and biases of the network only after the entire training set, that is, input vectors have been applied to the network. The gradients calculated at each training samples are added together to determine the change in weights and biases. Traingd function is used for the training of the network. Triangd is network training function 
that updates weight and bias values according to gradient descent. The network is created using newff. The newff creates feedforward back-propagation network (MATLAB, 2007).

\section{SYNTAX}

net $=$ newff(PR, $[\mathrm{SI}, \mathrm{S} 2, \ldots \mathrm{Sn}]\{\mathrm{TFI}$ TF2 $\ldots \mathrm{TFN}\}, \mathrm{BTF}$, $\mathrm{BLF}, \mathrm{PF})$

newff takes several arguments.

$\mathrm{PR}=\mathrm{R} \times 2$ matrix of min and max values for $\mathrm{R}$ input elements $\mathrm{Sl}=$ size of ith layer, for N1-layer

TF 1 - Transfer function of first layer (tansig)

TF2 - Transfer function of second layer (log sig)

TF3 - Transfer function of third layer (purelin)

BTF - Back propagation network training function (traingd)

BLF (Backpropagation weight / bias learning function (learngdm) and returns $\mathrm{N}$-layer feedforward backpropagation network.

The connection of the input to hidden layer, then the first hidden layer to output layer is automatically achieved when newff function is called. And as each layer has its own transfer function, the newff provides a means of specifying the transfer function of the layers in its syntax. The following are the seven parameters associated with traingd:

Epochs, show, goal, time, min-grad, max-fail and Ir

The Neural Network Multilayer Perceptron (NNMP) uses the tansig and logsig for the first and second hidden layers. The aim of using these is to get outputs in those two hidden layers of values between -1 and 1 . But since the target values the NNMP is created to approximate are greater than the range of values between -1 and 1 , Purelin is used at the output layer. This enables the network to output values of any magnitude.

\subsubsection{System Model}

Considering the input variables, the following system model was considered for the performance evaluation of academic staff of tertiary institutions.

$P E=F(Q J P, Q B P, Q C P, Q P S, R U N, I F R, P S T, P C S, P P A$, CVR, ACE, RST, SEI, PSE, PGI)

The input-output schematic of the system is shown in Figure 4 below.

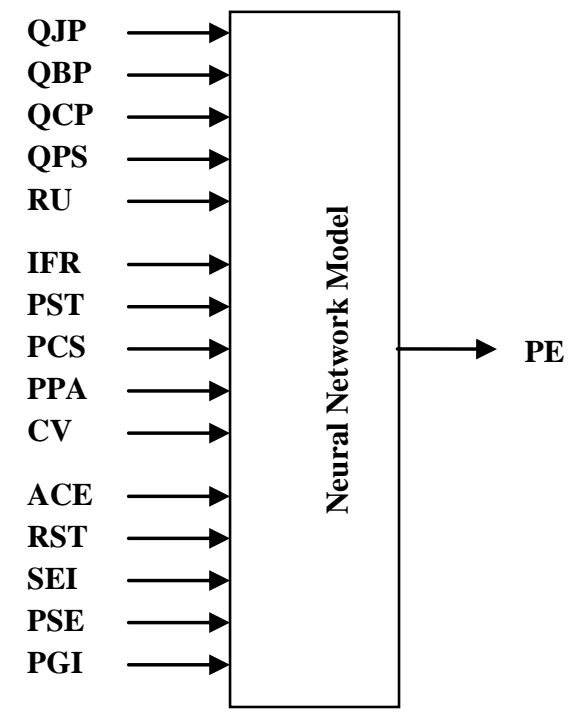

Figure 4: Input-Output schematic of system
KEY

$\mathrm{QJP}=\underline{\mathrm{Kuality}}$ of journal publication

$\mathrm{QBP}=$ Quality of book publications

$\mathrm{QCP}=$ Quality of conference proceedings

QPS = Quality of undergraduate Supervision

RUN = Relevance of research to the needs of the university

IFR =.Industrial focus of research

PST $=$ Performance of students in industrial training

$\mathrm{PCS}=$.Performance in community service

PPA $=$ Performance in professional associations

$\mathrm{CVR}=$ Commercial viability of research

$\mathrm{ACE}=$ Academic experience

RES = Relevance of specialization to courses taught

SEI $=$. Student Evaluation of Instructor

PSE $=$.Performance of students in examinations

PGI $=$ Performance of graduates in the industry

\subsubsection{Training Of The Neural Network}

The Neural Network system model was simulated using Matrix Laboratory Package and Java also trained using the 150 cases of the dataset. Figure 5 below illustrates with a flowchart the procedure adopted in the training of the Neural Network.

\subsubsection{Algorithm For MPNN}

// Assemble the training Data

1. Initialize the weights to inputs of hidden layer 1

2. Multiply the input vectors with their connecting weights

3. Compute the total weighted input

4. Threshold the total weighted input by transig to get output for the first hidden layer.

5. Propagate output of the first hidden layer input of the second hidden layer.

6. Initialize the weights that connect hidden layer 1 to hidden layer 2.

7. Repeat step 2 and 3

8. Threshold the total weighted input by logsig to get output and input for the second hidden layer and output layer respectively.

9. Initialize the weights that connect hidden layer 2 to output layer.

10. Repeat step 2 and 3 to get the output values.

11. Threshold the total weighted output by purelin to get the actual output values.

12. If the output values are equivalent to the target values Then

13. Go To Stop

Else

12. Compute EA // EA is the difference between the actual // values and the target values.

13. Convert EA to E1 // E1 is the rate at which error changes as the total input received by a unit is changed.

14. Compute EW // EW is the error derivatives of the weights. //That is how the error changes as each weight is increased or // decreased slightly.

15. Multiply those EAs of those output units and add the products.

16. Compute EAs for other layers by repeating Step 12 to 15 // Moving from layer to layer in a direction opposite the way activities // propagate.

17. Repeat 2 to 13

18. Stop 


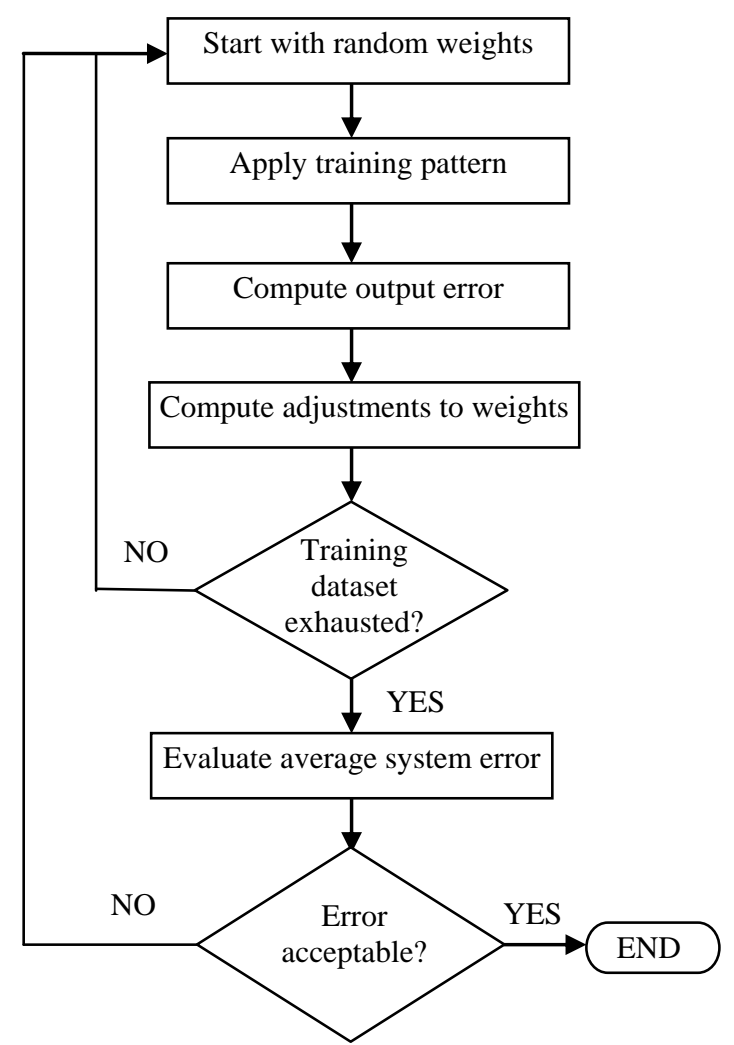

Figure 5: Training procedure for the multi-layer preceptron

\subsubsection{The Performance of the Model}

The performance of NN model is to a large degree dependent on the data on which they are trained and, looking at the performance result in Table 7 below, the parameters used in all the factors are shown in the Table. The learning parameters for the training and testing of the survey data varies from 0.07 to 0.1 and the momentum parameter approaches to zero value ( 0.01 to 0.03$)$. The number of cycles varies in different models for various factors. The value ranges from 293380 to 21775 for factor 1 . The percentage of correct output is decided by considering the lowest root mean square error (RMSE). The values of correct outputs for different factors for P-E (performance Evaluation) model are found to be $77 \%, 90 \%$ and $70 \%$.
Table 7: The System Performance of the model

\begin{tabular}{|c|c|c|r|c|c|}
\hline $\begin{array}{c}\text { Neural } \\
\text { Networ } \\
k\end{array}$ & $\begin{array}{c}\text { Learning } \\
\text { Paramet } \\
\text { Models }\end{array}$ & $\begin{array}{c}\text { Momentu } \\
\text { er }\end{array}$ & $\begin{array}{c}\text { Numbe } \\
r \text { of } \\
r\end{array}$ & $\begin{array}{c}\text { RM } \\
\text { Cycles } \\
\text { erro } \\
r\end{array}$ & $\begin{array}{c}\text { Percenta } \\
\text { ge of } \\
\text { Correct } \\
\text { Output }\end{array}$ \\
\hline QJP & 0.10 & 0.02 & 293 & 0.21 & $77^{*}$ \\
\hline QBP & 0.09 & 0.03 & 18680 & 0.22 & 62 \\
\hline QCP & 0.08 & 0.01 & 461 & 0.25 & 69 \\
\hline PST & 0.09 & 0.02 & 379 & 0.21 & 69 \\
& & & 195 & & \\
\hline QPS & 0.07 & 0.01 & 21775 & 0.15 & $90^{*}$ \\
\hline IFR & 0.09 & 0.03 & 30855 & 0.15 & 60 \\
\hline RUN & 0.08 & 0.01 & 17725 & 0.17 & 70 \\
\hline PCS & 0.10 & 0.03 & 3730 & 0.19 & 60 \\
\hline CVR & 0.08 & 0.02 & 4150 & 0.15 & $70^{*}$ \\
\hline PPA & 0.08 & 0.01 & 7975 & 0.17 & 70 \\
\hline ACE & 0.09 & 0.03 & 6500 & 0.18 & 70 \\
\hline RST & 0.10 & 0.02 & 3980 & 0.19 & 69 \\
\hline SEI & 0.09 & 0.03 & 7095 & 0.07 & $70^{*}$ \\
\hline PGI & 0.09 & 0.01 & 3350 & 0.08 & 70 \\
\hline PSE & 0.09 & 0.03 & 7320 & 0.07 & 50 \\
\hline
\end{tabular}

$*$ indicate the highest percentage of correct output

\subsubsection{Comparison of Models}

To compare the models with more conventional techniques parallel experiments were performed with the same data sets. Looking at the above Table 7 and comparing it with the multivariate model result produced in Table 5 and Table 1 . Each of the above models for a particular factor was run varying the learning parameter, momentum parameter and number of cycles till RMSE is minimized.

A model is said to perform best when the percentage of correct outputs is higher for the same RMS value. The learning parameters lay between 0.07 and 0.1 , whereas the momentum parameter approached zero (0.01 to 0.03$)$. The number of cycles varied from model to model for different Tertiary Institutions Policies. The value ranged from 293380 to 21775 for Factor 1, whereas it ranged from 6500 to 7320 for Factor 2. But for Factor 3, the training cycles range from 2760 to 7975 . The RMSE ranged from 0.15 to 0.25 for Factorl whereas it ranged from 0.07 to 0.08 for Factor 3.

Considering the maximum percentage of correct outputs with minimum RMSE, the P-E MPNN model was found to be the best model for predicting correct output for the academic staff of tertiary institutions. The values of correct outputs for different factors for the P-E model were found to be $90 \%$, $77 \%$ and $70 \%$ for Factor1, 2, 3 respectively. The parameters used in the models are shown in Table 7.

As a matter of fact, the comparative study of the models suggests that the P-E model has better predictive power for the academic staff promotion evaluation exercise in tertiary institutions. That is to say that research has the highest contribution to the productivity of the institutions. Since statically evidence also favours this model, it will be used for predicting service quality in tertiary institutions and identifying deficiencies in the system according to the factors classified and studied under this research. 


\section{CONCLUSION}

This study presents the prediction of performance evaluation using Neural Network model. The independent variables were principal components from 15 Human Resource Management Metrics. The results presented above shows that these independent variables appear to be useful in predicting performance evaluation effectively.

The Neural Network model demonstrated that they were able to provide an adequate model for predicting performance evaluation effectively. The performance of Neural Network model is to a large degree dependent on the data on which they are trained, and the availability of suitable system data determines the extent to which performance evaluation models are to be developed

The results arrived at give indications that there are some innovations that can still be introduced to make the model accommodate other staff of the tertiary institutions; administrative and technical staff.

\section{FUTURE STUDY}

i. The model can be expanded in future to include the evaluation of other categories of the tertiary institutions staff; administrative and technical staff.

ii. A search for a complete set of variables that integrates academic, administrative and technical staff is desirable, the establishment of workable models for their effective implementation.

iii. Determination of what should be the appropriate range for selection and promotion of workers because promotion policies of various institutions may differ depending on their goals and objectives. This will help find the right man for the right job; (unless the right peg is put into the right hole, the growth of the tertiary institutions will be stunted)

\section{REFERENCES}

[1] Leap T.L and Crino M.D, (2007): Personnel / Human Resource Management. Macmillan Publishing Company.

[2] [Uzoka and Akinyokun, (2005): Knowledge Based System for Human Resource Evaluation in a University
Environment, Journal of Testing and Evaluation, Vol. 33, No.1

[3] Liu P.H and Wei C.C, (2004): A group Decision Making Technique for Appraising the Performances of Organization. International Journal of the Computer, the Internet and Management Vol.8, No2, Pages 39-49. Taiwan.

[4] Saaty T.L, and Vargas L.G, (2004): Diagnosis with Dependent Symptoms: Baye's Theorem and Analytic Hierarchy Process; Operation Research. Vol.46, Issue 4, Pages 491-503.

[5] Akinyokun,O.C(2002):Neuro-Fuzzy Expert System for Human Resource Perform Evaluation, First Bank of Nigeria Plc Endowment Fund Public lecture, Series 1, Federal University of Technology, Akure, Nigeria.

[6] Marengo et al (2007): Principal Component Analysis: Neural Networks Architectures for content addressable Memory: IEE Proceedings P.138, 33-39.

[7] [Massart et al (2000): Multivariate Statistical Computation Analysis. Published by John Wiley and sons Inc.

[8] Vandeginste et al, (2000): Multivariate Modeling Published by John Wiley and sons Inc.

[9] Garcia de Ceca and Moro, (2000): Neural Network Approach; Pages 330-340.

[10] Sarhan and Helalat, (2007): Error Propagation. MIT Press, Cambridge MA, Chapters 10.

[11] Akinyokun, O.C and Uzoka F.M (2007): Factor Analysis of the effects of Academic Staff Profile on the investment Portfolio of a university, International Journal of the Computer, the Internet and Management 15 (1), 51-62. (11)

[12] Aggarwal et al (2006): Novelty detection and Neural Networks Validation.

[13] Hansen (2001): Using Neural Network to Forecast Automobiles Sales. The twenty third annual Pittsburgh conferences on Modeling and Simulation, University of Pittsburgh, U.S.A. 